\title{
Unicornuate Uterus with Rudimentary Horn as a Rare Etiology of Secondary Dysmenorrhea: A Case Report
}

\author{
Zuhdi Khalid Nagshabandia Bindu Isaac ${ }^{a}$ Inshia Begum ${ }^{b}$ \\ a Obstetrics and Gynecology, Latifa Hospital, Dubai Health Authority, Dubai, United Arab Emirates; 'bnternship, \\ Medical Education and Research Department, Dubai Health Authority, Dubai, United Arab Emirates
}

\section{Keywords}

Unicornuate uterus · Noncommunicating rudimentary horn · Congenital uterine anomaly · Magnetic resonance imaging · Dysmenorrhea

\section{Abstract}

Congenital uterine anomalies are an uncommon type of female genital malformations caused by abnormal development of müllerian ducts during embryogenesis. Patients with an obstructive uterine anomaly have a higher risk of developing gynecological and obstetric complications that may present at menarche or later in life. We present a case of severe dysmenorrhea in a young teenager caused by obstructive hematometra in a noncommunicating horn of the unicornuate uterus. A differential diagnosis of a possible anomaly was made using 2-dimensional pelvic ultrasonography, which was later confirmed using MRI that revealed an anomalous uterine cavity with a single left-sided cornua communicating with the cervix and a distended right-sided rudimentary horn. She underwent a right salpingectomy with rudimentary horn excision, which was successfully managed laparoscopically. This case emphasizes the importance of physi- cians being cognizant in identifying patients with uterine anomaly to provide appropriate treatment and prevent adverse reproductive outcomes.

(C) 2021 The Author(s)

Published by S. Karger AG, Basel

\section{Introduction}

Congenital uterine anomalies (CUAs) result from an abnormal formation, fusion, or resorption of the müllerian ducts during fetal life. According to a meta-analysis, the overall prevalence of CUAs is $5.5 \%$, with unicornuate uterus accounting for $0.1 \%$ of the population, amongst which $0.5 \%$ were infertile, $0.5 \%$ suffered miscarriage, and $3.1 \%$ of women had both miscarriage and infertility [1]. The clinical spectrum of unicornuate uterus can vary from an asymptomatic and incidental finding to complex reproductive pathology often leading to subfertility and miscarriages [2]. One of the most common symptoms of CUAs is dysmenorrhea or menstrual cramps which also happens to be a frequent complaint among adolescent females attending the outpatient department [3]. Secondary dysmenorrhea, which is usually pathological, should always raise the suspicion of uterine malformation that

C 2021 The Author(s).

Published by S. Karger AG, Basel

This is an Open Access article licensed under the Creative Commons Attribution-NonCommercial-4.0 International License (CC BY-NC) (http://www.karger.com/Services/OpenAccessLicense), applicable to the online version of the article only. Usage and distribution for commercial purposes requires written permission.
Correspondence to:

Bindu Isaac, binduisaacp@yahoo.co.in 
Fig. 1. MRI pelvis with contrast demonstrating unicornuate uterus on the left (yellow arrow) and a functional noncommunicating rudimentary horn containing hematometra on the right (white arrow). Coronal view (a) and axial view (b).
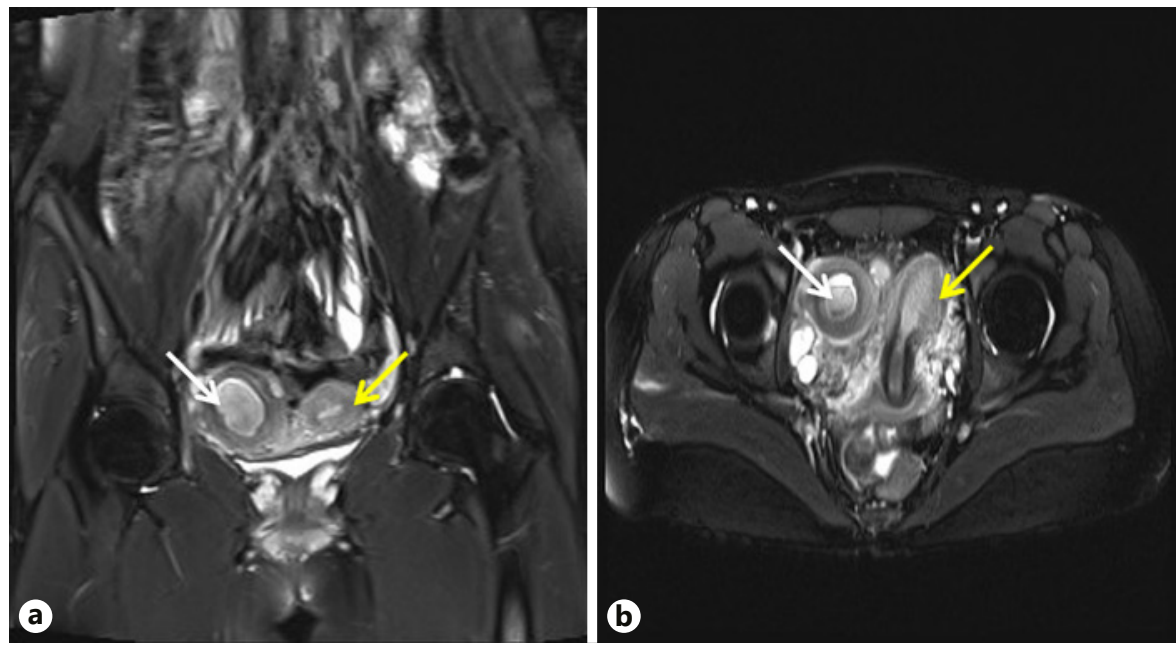

necessitates investigation and treatment. Owing to the wide variety of presentations, this clinical condition remains an interesting field of study with regard to its diagnosis and challenges in its management.

\section{Case Presentation}

We present the case of a 16-year-old nulligravid, who presented to the emergency department of a tertiary care hospital with complaint of severe dysmenorrhea. She had attained menarche at the age of 13 years and reported progressive dysmenorrhea for the last 18 months. The pain was cramping in nature persisting for a week, even after the menstrual bleeding subsided and was minimally relieved with analgesics. It was associated with constipation and increased urinary frequency that started a week prior to presentation. She had regular menstrual cycles and was not sexually active. She had no prior significant medical or surgical history.

On admission, she had hemodynamically stable vitals. Abdominal examination revealed notable tenderness in the suprapubic and right iliac fossa region with absence of guarding. Systemic examination otherwise was unremarkable.

Transabdominal ultrasonography revealed a pelvic mass in the right adnexal region with walls being isoechoic to the myometrium and contained hyperechoic material. Subsequent MRI revealed an anomalous uterine cavity with a single left-sided cornua communicating with the cervix, pushed to the left by a distended rightsided rudimentary horn with normal myometrial signal intensity. Intracavitary fluid with high $\mathrm{T} 1$ and $\mathrm{T} 2$ signal intensity indicated hematometra in the rudimentary horn with no free fluid in the pelvis (Fig. 1).

After detailed discussion with the patient and her family about the surgery, an informed consent was obtained and a therapeutic laparoscopic evaluation under general anesthesia was performed. The exploratory findings were consistent with the MRI study, which showed a noncommunicating rudimentary horn measuring $4 \times 4 \mathrm{~cm}$ with its connecting fallopian tube, suspended by a fibrous

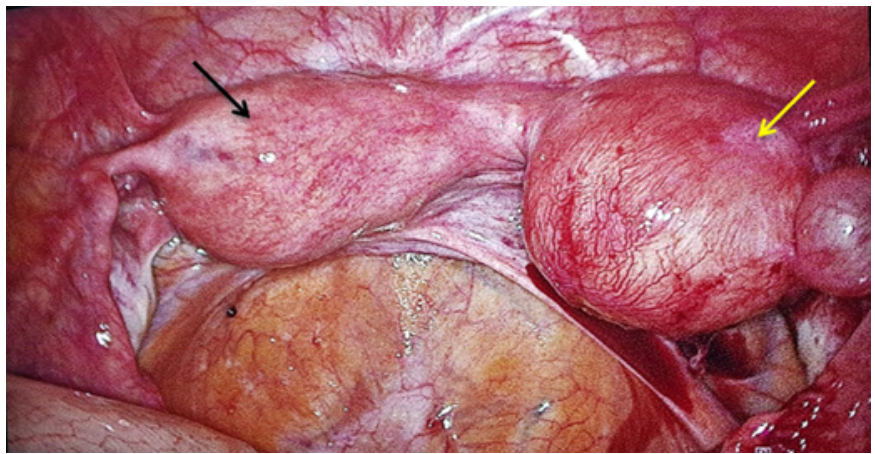

Fig. 2. Laparoscopy view showing a noncommunicating rudimentary horn on the right with its fallopian tube and ovary (black arrow), suspended by a fibrous band connecting to the unicornuate uterus on the left (yellow arrow).

band attached to the right of the uterus (Fig. 2). Both tubes and ovaries were normal in shape and size.

Initially, the round ligament, the ovarian ligament, and anterior leaflet of the broad ligament ipsilateral to the rudimentary horn were cauterized and cut. A right salpingectomy was done at the cornual end. Following dissection into the retroperitoneal space, a branch of the right uterine artery supplying the rudimentary horn was coagulated. The fibromuscular connection was cut, the rudimentary horn was incised draining altered dark blood, and was later morcellated. The pieces of the horn along with the connecting tube were removed and sent for histopathology evaluation, which revealed myometrium with multiple adenomyosis foci. The postoperative period was uneventful, and the patient was discharged 3 days later. She was followed up in the gynecology clinic after a 2 -month period and reported less dysmenorrhea. 
Table 1. The American Fertility Society classification of unicornuate uterus

Type A: with rudimentary horn

A1: rudimentary horn containing endometrium

A1a: communicating (10\%)

A1b: noncommunicating (22\%)

A2: rudimentary horn with no endometrial cavity (33\%)

Type B: no rudimentary horn (35\%)

\section{Discussion}

The female reproductive organs develop from paired müllerian (paramesonephric) ducts at the sixth week of gestation [4], which fuses to form the uterus, cervix, and upper two-thirds of the vagina. CUAs result from a failure of fusion, abnormal development, or incomplete resorption of the müllerian ducts. The sporadic nature of the majority of these anomalies, on the other hand, may indicate a multifactorial etiology involving extrauterine and intrauterine environmental factors such as infections, ionizing radiation, or teratogenic drugs (e.g., thalidomide and diethylstilbestrol) during early pregnancy [5]. We believe our case is congenital as there was no significant prenatal history to suggest an acquired reason for the malformation.

CUAs are classified into a 7-class system, with our patient presenting with the class 2 - unicornuate uterus. Unicornuate uterus results from an abnormal development of one of the paired müllerian ducts [6]. According to the American Fertility Society [7], it can be classified further into the subtypes as given in Table 1.

Patients with this uterine anomaly are more likely to experience gynecological problems such as hematometra, hematosalpinx, endometriosis, dysmenorrhea, chronic pelvic pain, and primary infertility, as well as obstetric complications like miscarriages, ectopic pregnancies, rupture of the uterus, and preterm labor [8]. With regard to this case, the patient presented with increasing postmenstrual pain, urinary frequency, and constipation, which we believe was due to distension of the rudimentary horn owing to its functional endometrium and its pressure effect on neighboring organs.

Precise assessment of the internal and external contours of the uterus is essential in diagnosing and classifying CUAs accurately. Previously, the combination of laparoscopy and hysteroscopy was the gold standard modality; however, imaging studies such as ultrasonography, hysterosalpingography (HSG), sonohysterography, and MRI are less invasive and are used to screen, diagnose, and classify CUAs [1]. Although standard 2-dimensional transvaginal scanning (TVS) and HSG are good screening tools for uterine anomalies, 3-dimensional TVS and MRI can precisely classify CUAs $[9,10]$. Since TVS and HSG cannot be carried out in females with intact hymen, an MRI was performed on this patient.

Rudimentary horn and its liability for ectopic pregnancy has been described in multiple literature studies as being presumably due to intraperitoneal sperm or oocyte migration $[11,12]$ and were associated with higher incidence of abortion or rupture of the horn, especially in the second and third trimesters [13]. Furthermore, considering the fact that hematometra, hematosalpinx, and endometriosis are consequences of retrograde menstruation [14], the rudimentary horn and its connecting fallopian tube were removed during laparoscopy for this patient. Our goal was to perform a reconstructive surgery to restore the structural and functional integrity and to alleviate symptoms via minimally invasive procedure.

Although unicornuate uterus is often associated with renal anomalies, occurring in $40.5 \%$ of cases [15], with renal agenesis being the most common, recorded in $67 \%$ of cases [16], our patient had no such abnormalities. An MRI should be used in these patients' diagnostic workup to outline the rudimentary horn variant and to assess the presence of associated renal anomalies.

\section{Conclusion}

A case of rudimentary uterus is clinically misleading, and therefore an appropriate diagnostic method is needed for prompt diagnosis. Although there is no one specific symptom for clinically diagnosing uterine anomaly, one should have a high level of suspicion when a young female patient presents with dysmenorrhea that does not respond to analgesics. Only imaging, such as transvaginal ultrasound and MRI, can provide a definitive diagnosis, which is useful not only for diagnosing but also for surgical planning. This literature also explains the significance of laparoscopic removal of rudimentary horn and its connecting fallopian tube as the best treatment option, particularly for younger women in their fertile age, as in our case to prevent future gynecological and pregnancy-related complications.

\section{Statement of Ethics}

Signed consent was obtained from the patient's family for publication of this case report and the associated images. According to the journal's criteria, ethical approval is mandated only for research studies, while patient consent is sufficient for case reports.
Nagshabandi/Isaac/Begum 


\section{Conflict of Interest Statement}

The authors have no conflicts of interest to declare.

\section{Funding Sources}

There were no funding sources.

\section{Author Contributions}

All authors contributed equally to this report.

\section{Data Availability Statement}

All data generated or analyzed during this study are included in this article. Further enquiries can be directed to the corresponding author.

\section{References}

1 Chan YY, Jayaprakasan K, Zamora J, Thornton JG, Raine-Fenning N, Coomarasamy A. The prevalence of congenital uterine anomalies in unselected and high-risk populations: a systematic review. Hum Reprod Update. 2011;17(6):761-71.

2 Akhtar MA, Saravelos SH, Li TC, Jayaprakasan K. Reproductive implications and management of congenital uterine anomalies: scientific impact paper no. 62 November 2019. BJOG. 2019;127(5):e1-13.

3 Borah T, Das A, Panda S, Singh S. A case of unilateral dysmenorrhea. J Hum Reprod Sci. 2010;3(3):158-9.

4 Thapa S, Mohammed A, Chowdhury M, Mensah S. Case report on an unusual presentation of rudimentary uterus. J Univers Surg. 2015;3(3):14. (ISSN 2254-6758).

5 Hassan MA, Lavery SA, Trew GH. Congenital uterine anomalies and their impact on fertility. Womens Health. 2010 May;6(3):443-61.

6 Marcal L, Nothaft MA, Coelho F, Volpato R, Iyer R. Mullerian duct anomalies: MR imaging. Abdom Imaging. 2011;36(6):756-64.
7 Khati NJ, Frazier AA, Brindle KA. The unicornuate uterus and its variants: clinical presentation, imaging findings, and associated complications. J Ultrasound Med. 2012;31(2): 319-31.

8 Reichman D, Laufer MR, Robinson BK. Pregnancy outcomes in unicornuate uteri: a review. Fertil Steril. 2009;91(5):1886-94.

9 Grimbizis GF, Di Spiezio Sardo A, Saravelos $\mathrm{SH}$, Gordts S, Exacoustos C, Van Schoubroeck D, et al. The Thessaloniki ESHRE/ ESGE consensus on diagnosis of female genital anomalies. Gynecol Surg. 2015;13(1):1-16.

10 Szkodziak P, Woźniak S, Czuczwar P, Paszkowski T, Milart P, Wozniakowska E, et al. Usefulness of three dimensional transvaginal ultrasonography and hysterosalpingography in diagnosing uterine anomalies. Ginekol Pol. 2014;85(5):354.

11 Arslan T, Bilgiç E, Sentürk MB, Yücel N. Rudimentary uterine horn pregnancy: a mystery diagnosis. Fertil Steril. 2009;92(6):2037-3.
12 Scheibner K, Kuhn A, Raio L, Brühwiler H, Müller MD. The non-communicating rudimentary horn: diagnostic and therapeutic challenges. Gynecol Surg. 2007;4(3):207-11.

13 Mengistu K, Bobe T, Tilahun G, Kifle K, Geleta D. Rudimentary horn pregnancy diagnosed after laparotomy. Case Rep Obstet Gynecol. 2020;2020:5816487.

14 Obeidat RA, Aleshawi AJ, Tashtush NA, Alsarawi $\mathrm{H}$. Unicornuate uterus with a rudimentary non-communicating cavitary horn in association with VACTERL association: case report. BMC Womens Health. 2019; 19(1):71.

15 Reichman D, Laufer MR, Robinson BK. Pregnancy outcomes in unicornuate uteri: a review. Fertil Steril. 2009;91(5):1886-94.

16 Humane A, Agrawal N. Unicornuate uterus with noncommunicating functional horn: a case report [Internet]. Int J Curr Res. 2020; 12(3):10845-7. Available from: http://journalcra.com/article/unicornuate-uterus-noncommunicating-functional-horn-case-report. 